\title{
A COMPARISON OF DUAL LAGRANGE MULTIPLIER SPACES FOR MORTAR FINITE ELEMENT DISCRETIZATIONS
}

\author{
BARBARA I. WOHLMUTH ${ }^{1}$
}

\begin{abstract}
Domain decomposition techniques provide a powerful tool for the numerical approximation of partial differential equations. We focus on mortar finite element methods on non-matching triangulations. In particular, we discuss and analyze dual Lagrange multiplier spaces for lowest order finite elements. These non standard Lagrange multiplier spaces yield optimal discretization schemes and a locally supported basis for the associated constrained mortar spaces. As a consequence, standard efficient iterative solvers as multigrid methods or domain decomposition techniques can be easily adapted to the nonconforming situation. Here, we introduce new dual Lagrange multiplier spaces. We concentrate on the construction of locally supported and continuous dual basis functions. The optimality of the associated mortar method is shown. Numerical results illustrate the performance of our approach.
\end{abstract}

Mathematics Subject Classification. 65N30, 65N55.

Received: July 12, 2001.

\section{INTRODUCTION}

Nonconforming domain decomposition techniques provide a more flexible approach than standard conforming formulations. They are of special interest for time dependent problems, rotating geometries, diffusion coefficients with jumps, problems with local anisotropies, corner singularities, and when different terms dominate in different regions of the simulation domain. Very often heterogeneous problems can be decomposed into homogeneous subproblems for which efficient discretization techniques are available. To obtain a stable and optimal discretization scheme for the global problem, the information transfer and the communication between the subdomains are of crucial importance. We present different approaches within the framework of mortar methods $[5,6]$. Originally introduced as a domain decomposition method for the coupling of spectral elements, these techniques are nowadays used in a large class of nonconforming situations. Thus, the coupling of different physical models, discretization schemes, or non-matching triangulations along interior interfaces of the domain can be analyzed by mortar methods. The paper is organized as follows: in the rest of this section, we give a brief introduction to mortar finite element methods. We introduce in Section 2 new Lagrange multiplier spaces. In contrast to earlier approaches, the introduced Lagrange multiplier basis functions are continuous and biorthogonal to the standard hat functions. Different examples are given in $2 \mathrm{D}$ and in $3 \mathrm{D}$. The optimality of the associated discrete mortar formulation is shown in Section 3. A priori bounds for the $L^{2}$-norm and the energy

Keywords and phrases. Mortar finite elements, dual space, non-matching triangulations, multigrid methods.

1 Math. Institut, Universität Stuttgart, Pfaffenwaldring 57, 70569 Stuttgart, Germany.

e-mail: wohlmuth@mathematik.uni-stuttgart.de 
norm are established. In Section 4, we present numerical results for different dual Lagrange multiplier spaces illustrating the performance of the nonconforming approach. In particular, we consider the discretization error in the $L^{2}$-norm, the energy norm and in a weighted $L^{2}$-norm for the Lagrange multiplier.

We consider the following elliptic second order boundary value problem

$$
\begin{aligned}
-\operatorname{div}(a \nabla u)+b u & =f, & & \text { in } \Omega, \\
u & =0, & & \text { on } \partial \Omega .
\end{aligned}
$$

Here, $0<a_{0} \leq a \in L^{\infty}(\Omega), f \in L^{2}(\Omega), 0 \leq b \in L^{\infty}(\Omega)$, and $\Omega \subset \mathbb{R}^{d}, d=2,3$, is a bounded polygonal domain. Let $\Omega$ be decomposed into $K$ non-overlapping polyhedral subdomains $\Omega_{k}$ such that $\bar{\Omega}=\bigcup_{k=1}^{K} \bar{\Omega}_{k}$. We restrict ourselves to the geometrical conforming situation where the intersection between the boundaries of any two different subdomains $\partial \Omega_{l} \cap \partial \Omega_{k}, k \neq l$, is either empty, a vertex, a common edge or face in 3D. Geometrically nonconforming situations are technically more difficult to handle. A possibility to reduce these complications is to require that each vertex of the decomposition is also a vertex of each adjacent triangulation.

We define on each subdomain a simplicial or hexahedral triangulation $\mathcal{T}_{k: h_{k}}$, the meshsize of which is bounded by $h_{k}$. The discrete space of conforming piecewise linear, bilinear or trilinear finite elements on $\Omega_{k}$ associated with $\mathcal{T}_{k ; h_{k}}$, which satisfies homogeneous Dirichlet boundary conditions on $\partial \Omega \cap \partial \Omega_{k}$, is denoted by $X_{h_{k}}$. Then, the mortar method is characterized by the introduction of a discrete Lagrange multiplier space $M_{h_{m}}\left(\gamma_{m}\right)$ on the interfaces $\gamma_{m}, 1 \leq m \leq M$, of the decomposition. For each interface, there exists a couple $1 \leq l(m)<k(m) \leq K$ such that $\bar{\gamma}_{m}=\partial \Omega_{l(m)} \cap \partial \Omega_{k(m)}$. Moreover, each interface $\gamma_{m}$ is associated with a $(d-1)$-dimensional mesh $\mathcal{S}_{m ; h_{m}}$, inherited from either $\mathcal{T}_{k(m) ; h_{k(m)}}$ or $\mathcal{T}_{l(m) ; h_{l(m)}}$. The choice is arbitrary but should be fixed. In general, these triangulations do not coincide. The elements of $\mathcal{S}_{m ; h_{m}}$ are boundary edges in 2D and boundary faces in 3D of either $\mathcal{T}_{l(m) ; h_{l(m)}}$ or $\mathcal{T}_{k(m) ; h_{k(m)}}$. The subdomain from which the interface inherits its triangulation is called slave or non-mortar side, the opposite one master or mortar side.

To obtain the mortar approximation $u_{h}$, as a solution of a discrete variational problem, there are two main approaches. The first one has been introduced in $[5,6]$ and gives rise to a positive definite nonconforming variational problem. It is defined on a subspace $V_{h}$ of the product space $X_{h}:=\left\{v \in L^{2}(\Omega) \mid \quad v_{\left.\right|_{\Omega_{k}}} \in X_{h_{k}}, 1 \leq\right.$ $k \leq K\}$. The elements of $V_{h}$ satisfy weak continuity conditions across the interfaces. The constrained finite element space $V_{h}$ is given by

$$
V_{h}:=\left\{v \in X_{h} \mid \int_{\gamma_{m}}[v] \mu \mathrm{d} \sigma=0, \mu \in M_{h_{m}}\left(\gamma_{m}\right), 1 \leq m \leq M\right\} .
$$

Then, the nonconforming formulation of the mortar method can be given in terms of the constrained space $V_{h}$ : Find $u_{h} \in V_{h}$ such that

$$
a\left(u_{h}, v_{h}\right)=\left(f, v_{h}\right)_{0}, \quad v_{h} \in V_{h} ;
$$

see $[5,6]$. Here, the bilinear form $a(\cdot, \cdot)$ is defined as

$$
a(v, w):=\sum_{k=1}^{K} \int_{\Omega_{k}} a \nabla v \cdot \nabla w+b v w \mathrm{~d} x, \quad v, w \in \prod_{k=1}^{K} H^{1}\left(\Omega_{k}\right) .
$$

It is obvious that the quality of the nonconforming approach (1.2) and the properties of $V_{h}$ depend on the discrete Lagrange multiplier space $M_{h}:=\prod_{m=1}^{M} M_{h_{m}}\left(\gamma_{m}\right)$.

Let us consider the structure of $V_{h}$ in more detail. In general, $V_{h}$ is not a subspace of $H_{0}^{1}(\Omega)$ and thus $(1.2)$ is a nonconforming finite element method. Even for a nested sequence of global triangulations, the corresponding finite element spaces are non-nested. Secondly in general, no basis of $V_{h}$ with local support can be constructed. The constraints at the interfaces are given in terms of a $L^{2}$-orthogonality of the jumps, and an element $v_{h} \in X_{h}$ 


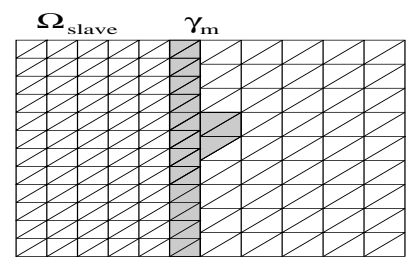

FigurE 1.1. Structure of the support of a nodal basis function in $V_{h}$, (standard).

belongs to $V_{h}$ if and only if

$$
\int_{\gamma_{m}}\left[v_{h}\right] \mu_{i} \mathrm{~d} \sigma=0, \quad 1 \leq i \leq n_{m}, 1 \leq m \leq M
$$

where $n_{m}:=\operatorname{dim} M_{h_{m}}\left(\gamma_{m}\right)$ and $\left\{\mu_{i}\right\}_{1 \leq i \leq n_{m}}$ defines a basis of $M_{h_{m}}\left(\gamma_{m}\right)$. We suppress the interface index $m$ in the case of the basis functions $\mu_{i}$. Following the approach in [3], the mortar method can equivalently be written as a saddle point formulation. Find $\left(u_{h}, \lambda_{h}\right) \in X_{h} \times M_{h}$ such that

$$
\begin{aligned}
a\left(u_{h}, v_{h}\right)-b\left(\lambda_{h}, v_{h}\right) & =\left(f, v_{h}\right)_{0}, & & v_{h} \in X_{h} \\
-b\left(\mu_{h}, u_{h}\right) & =0, & & \mu_{h} \in M_{h} .
\end{aligned}
$$

To obtain an order $h$ discretization scheme, the Lagrange multiplier space has to be chosen carefully. It has to be large enough to obtain a consistency error of order $h$. On the other hand, it has to be small enough to get a best approximation error of order $h$ and a uniform discrete inf-sup condition. A natural and efficient choice for the construction of good Lagrange multiplier spaces is to define the nodal Lagrange multiplier basis function locally and to associate them with the interior vertices of the slave side. In the following, we restrict ourselves to these situations. Now, we group the degrees of freedom of $X_{h}$ associated with the interface $\gamma_{m}$ into three groups $u_{\gamma_{m}}:=\left(u_{s}, u_{m}, u_{c}\right)$. Here, $u_{s}$ and $u_{m}$ are the nodal values of $u$ at the interior vertices of $\gamma_{m}$ on the slave and master side, respectively. The third group having the index ${ }_{c}$, represents all nodal values at the vertices on the boundary of $\gamma_{m}$. In $2 \mathrm{D}$, each interface not touching $\partial \Omega$ has two endpoints and thus $u_{c}$ has dimension four. In $3 \mathrm{D}$, the dimension depends on the mesh on the wirebasket. Then (1.3), can be written in its algebraic form as

$$
M_{s} u_{s}=-M_{m} u_{m}-M_{c} u_{c}
$$

where the entries of the mass matrices are given by $m_{i j}:=\int_{\gamma_{m}}\left[\varphi_{j}\right] \mu_{i} \mathrm{~d} \sigma$, and $\varphi_{j}$ corresponds to the different nodal basis functions on the slave and master side and associated with the vertices. The mass matrices are sparse due to the local structure of the supports of the involved basis functions. Formally, we can obtain the values on the slave side as $u_{s}=-M_{s}^{-1}\left(M_{m} u_{m}+M_{c} u_{c}\right)$. We note that $M_{s}$ is a square matrix whereas $M_{m}$ and $M_{c}$ are rectangular matrices. In general the inverse of the mass matrix $M_{s}$ is dense, and thus the values on the slave side depend globally on the values on the master side. Figure 1.1 shows the typical support of a basis function in $V_{h}$ associated with an interface, where the slave side is on the left.

The motivation of dual Lagrange multiplier spaces is based on the following observation. If $M_{s}$ in (1.5) is a diagonal matrix, then the values of $u_{s}$ depend locally on the values of the master side. Let us denote the nodal basis functions in $H_{0}^{1}\left(\gamma_{m}\right)$ associated with the $(d-1)$-dimensional mesh on the slave side by $\left\{\varphi_{i}\right\}_{1 \leq i \leq n_{m}}$. We call the basis functions $\left\{\mu_{i}\right\}_{1 \leq i \leq n_{m}}$ and $\left\{\varphi_{i}\right\}_{1 \leq i \leq n_{m}}$ biorthogonal and the Lagrange multiplier space dual if and only if

$$
\int_{\gamma_{m}} \mu_{i} \varphi_{j} \mathrm{~d} \sigma=c_{i} \delta_{i j} \int_{\gamma_{m}} \varphi_{j} \mathrm{~d} \sigma
$$




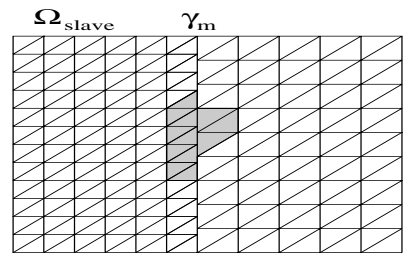

FiguRE 1.2. Structure of the support of a nodal basis function in $V_{h}$ (dual).

where $c_{i} \neq 0$. Without loss of generality, we can assume that $c_{i}=1$. Figure 1.2 depicts the structure of a constrained basis function if a dual Lagrange multiplier space is used.

\section{EXAMPles FOR DUAL LAGRANGe MULtiPlier SPACES}

In this section, we consider different biorthogonal basis functions. Dual Lagrange multiplier spaces have been successfully introduced for low order mortar finite elements in [13]. Recently, they have been generalized to higher order finite elements in [11]. We also refer to $[8,14]$ for a more detailed discussion and the construction in $3 \mathrm{D}$. In all our examples, the dual basis functions $\mu_{i}$ are associated with the interior vertices $p_{i}$ of $\mathcal{S}_{m ; h_{m}}$.

\subsection{The $2 \mathrm{D}$ case}

Here, we briefly review two discontinuous dual basis functions for $d=2$, and we refer to [14] for a numerical comparison of these Lagrange multiplier spaces with the standard one. We assume that the vertices are ordered lexicographically, and the two endpoints are denoted by $p_{0}$ and $p_{n_{m}+1}$. Figure 2.1 illustrates the interior dual basis functions, i.e., $2 \leq i \leq n_{m}-1$.
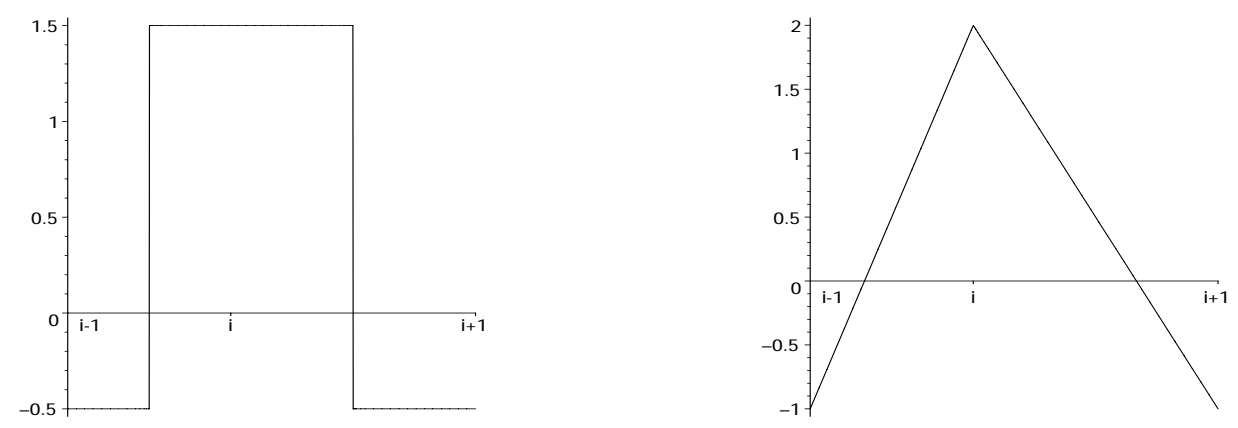

Figure 2.1. Discontinuous piecewise constant (left) and piecewise linear (right) dual basis functions.

On the left a piecewise constant dual basis function is shown, and on the right, a piecewise linear dual basis function is depicted. Both dual basis functions are discontinuous at the endpoints of their support. Although optimal a priori bounds are obtained and a locally supported basis for the constrained space $V_{h}$ exists, the use of these Lagrange multiplier spaces might be disadvantageous. In general, the numerical realization of the mortar method is based on the assembling of the entries of the mass matrix $M_{m}$ on $\gamma_{m}$

$$
m_{i j}:=-\int_{\gamma_{m}} \mu_{i} \varphi_{j}^{m} \mathrm{~d} \sigma,
$$

where $\varphi_{j}^{m}$ are the nodal basis functions on the master side. Then, the integration of a product of discrete functions living on two different meshes has to be carried out. One possible remedy might be to use quadrature rules, see [7]. The best approximation error requires a quadrature rule based on the slave side, whereas the 
consistency error requires the use of a quadrature rule based on the master side. We note that the discontinuities of the Lagrange multiplier basis functions are associated with the mesh on the slave side. Quadrature formulas applied to discontinuous functions might result in a considerable loss of accuracy if the quadrature nodes are not adapted to the discontinuities. Since we work with independent meshes on the master and the slave sides, the discontinuities of the Lagrange multiplier basis functions are in general not captured by quadrature points based on the master side. Therefore, it might be advisable to work with Lagrange multiplier basis functions which are continuous. Now, the idea is to modify appropriately the piecewise linear dual basis functions

$$
\mu_{i}:=\left\{\begin{array}{l}
2 \varphi_{i}-\varphi_{i-1}, x \in\left[p_{i-1}, p_{i}\right), \quad 2 \leq i \leq n_{m}-1, \\
2 \varphi_{i}-\varphi_{i+1}, x \in\left[p_{i}, p_{i+1}\right],
\end{array}\right.
$$

and $\mu_{1}=1=\mu_{n_{m}}$ restricted to $\left[p_{0}, p_{1}\right]$ and $\left[p_{n_{m}}, p_{n_{m}+1}\right]$, respectively. On $\left[p_{1}, p_{2}\right]$ and $\left[p_{n_{m}-1}, p_{n_{m}}\right], \mu_{1}$ and $\mu_{n_{m}}$ have the same structure as $\mu_{i}, 2 \leq i \leq n_{m}-1$. There are many possibilities to modify the basis functions. We are interested in Lagrange multiplier basis functions where the support is as small as possible. Thus, we assume that the modified basis functions $\hat{\mu}_{i}$ satisfy

$$
\begin{aligned}
& {[\mathrm{P} 0] \quad \hat{\mu}_{i} \text { is continuous, }} \\
& {[\mathrm{P} 1] \quad \operatorname{supp} \hat{\mu}_{i}=\operatorname{supp} \varphi_{i}^{s},} \\
& {[\mathrm{P} 2] \quad \sum_{i=1}^{n_{m}} \hat{\mu}_{i}=1} \\
& {[\mathrm{P} 3] \quad \int_{\gamma_{m}} \hat{\mu}_{i} \varphi_{j}^{s} \mathrm{~d} \sigma=\delta_{i j} \int_{\gamma_{m}} \varphi_{j}^{s} \mathrm{~d} \sigma} \\
& {[\mathrm{P} 4] \quad\left\|\hat{\mu}_{i}\right\|_{0} \leq C\left\|\varphi_{i}^{s}\right\|_{0},\left|\hat{\mu}_{i}\right|_{1} \leq C\left|\varphi_{i}^{s}\right|_{1}}
\end{aligned}
$$

where $\varphi_{j}^{s}$ are the nodal basis functions on the slave side. Moreover, we restrict ourselves to transfer and reflexion invariant modifications, i.e., the interior basis functions $\hat{\mu}_{i}, 2 \leq i \leq n_{m}-1$, have the same structure and $\hat{\mu}_{i}$ restricted to $\left[p_{i}, p_{i+1}\right]$ is obtained from $\hat{\mu}_{i+1}$ restricted to $\left[p_{i}, p_{i+1}\right]$ by reflexion at the midpoint of $\left[p_{i}, p_{i+1}\right]$. In a first step, we consider $[\mathrm{P} 0]-[\mathrm{P} 4]$ in more detail. Let $g \in H^{1}([0,1])$ such that

$$
\begin{array}{ll}
\text { [g0 }] & g(t)=-g(1-t), t \in[0,1], \\
\text { [g1] } & g(1)=1, \\
\text { [g2 }] & \int_{0}^{1} g(t) p(t) \mathrm{d} t=0 \text { for all affine functions } p \in P_{1}([0,1]) .
\end{array}
$$

Now, we define a modified basis function $\hat{\mu}_{i}$ in terms of $\mu_{i}$ and $g$. We denote by $g_{i}$ the affine transformation of $g$ onto the edge $e_{i}=\left(p_{i-1}, p_{i}\right)$, and define

$$
\hat{\mu}_{i}:=\mu_{i}+\left\{\begin{array}{rl}
-g_{i}, & x \in\left[p_{i-1}, p_{i}\right), \\
g_{i+1}, & x \in\left[p_{i}, p_{i+1}\right],
\end{array} \quad 2 \leq i \leq n_{m}-1,\right.
$$

and $\hat{\mu}_{1}=1=\hat{\mu}_{n_{m}}$ restricted to $\left[p_{0}, p_{1}\right]$ and $\left[p_{n_{m}}, p_{n_{m}+1}\right]$, respectively. On $\left[p_{1}, p_{2}\right]$ and $\left[p_{n_{m}-1}, p_{n_{m}}\right], \hat{\mu}_{1}$ and $\hat{\mu}_{n_{m}}$ have the same structure as the interior $\hat{\mu}_{i}, 2 \leq i \leq n_{m}-1$.

Lemma 2.1. The modified basis functions $\hat{\mu}_{i}$ defined by (2.1) satisfy the properties [P0]-[P4].

The properties $[\mathrm{P} 0]-[\mathrm{P} 4]$ follow by construction. Obviously, there are many choices for the definition of $g$. Here, we consider two possibilities in more detail. We start by defining $g$ as a polynomial satisfying [g0]-[g2]. It is easy to verify that the minimal degree of $g$ is equal three. A straightforward calculation shows that the unique $g \in P_{3}([0,1])$ which satisfies [g0]-[g2] is given by:

$$
g(t):=(2 t-1)(1-10 t(1-t)) .
$$

The corresponding modified dual basis functions are shown in Figure 2.2. 

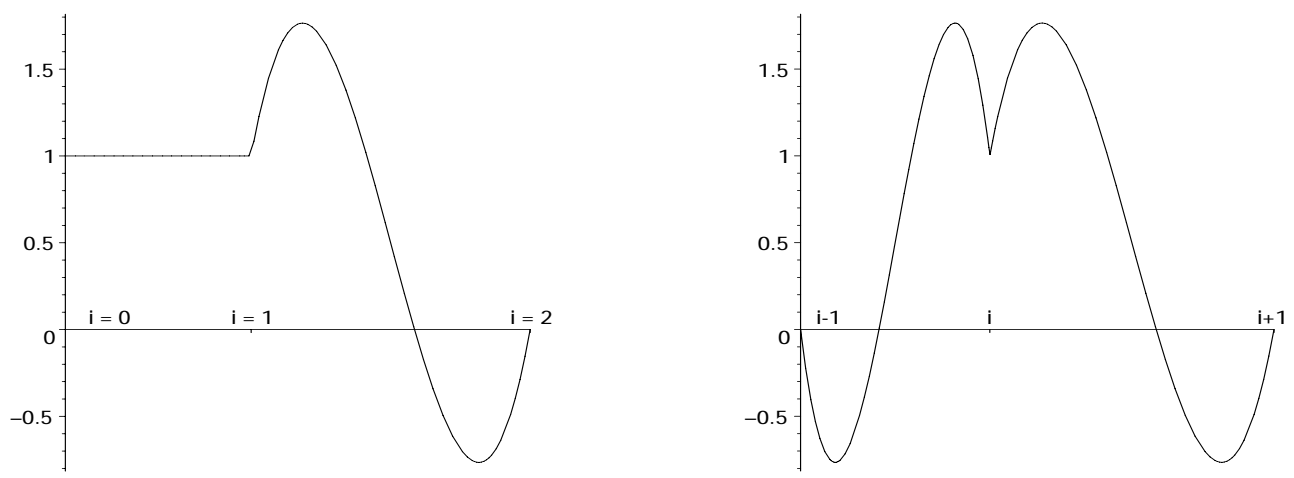

Figure 2.2. Continuous dual basis functions (piecewise cubic).
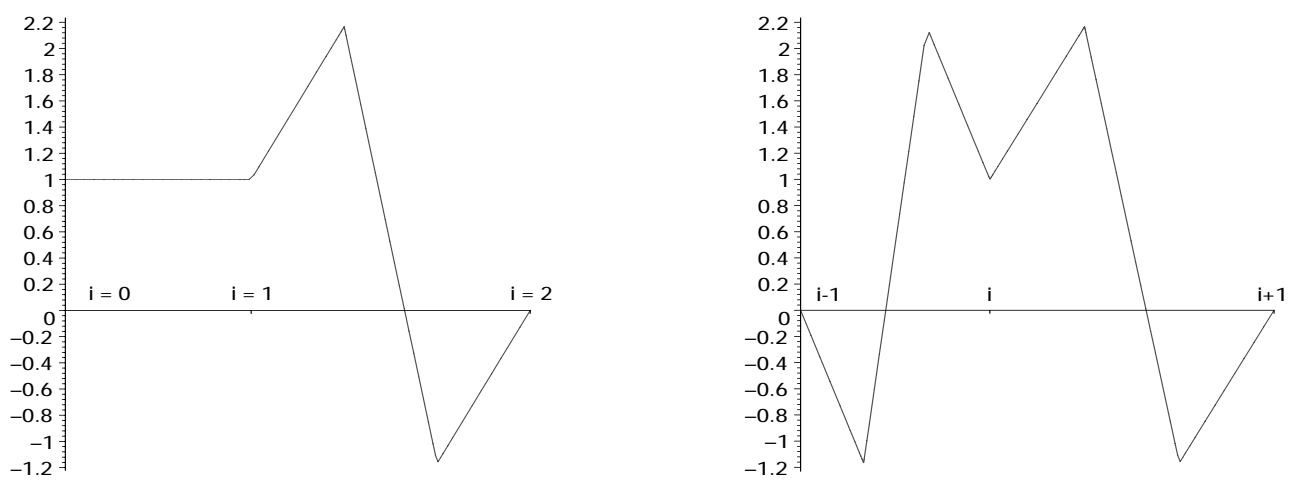

Figure 2.3. Continuous dual basis functions (piecewise linear).

A different possibility is to define $g$ as a piecewise affine function on $[0,1]$. Then, the conditions [g0]-[g2] yield that the unit interval has to be decomposed at least into three subintervals, e.g., we can define $g$ by

$$
g(t):= \begin{cases}-1+\frac{13}{2} t, & 0 \leq t<\frac{1}{3} \\ -7\left(t-\frac{1}{2}\right), & \frac{1}{3} \leq t \leq \frac{2}{3} \\ 1-\frac{13}{2}(1-t), & \frac{2}{3}<t \leq 1 .\end{cases}
$$

The associated dual basis functions are shown in Figure 2.3. Comparing Figure 2.2 and 2.3, we find that the two modified basis functions have a similar structure. Both have negative values close to the endpoints of the supports and values greater equal one in a neighborhood of the center vertex $p_{i}$.

We recall that both modified dual basis functions are obtained from the same piecewise affine dual basis function. The modifications are realized in terms of different $g$. The duality of the new basis functions follows by construction from [g2]. Figure 2.4 shows the two different choices for $g$.

\subsection{The $3 \mathrm{D}$ case}

In 3D, we consider the two different situations of rectangles and triangles on the interface. The hexahedral case can easily be handled. In that case, we use the tensor product structure, and we can obtain our dual basis 

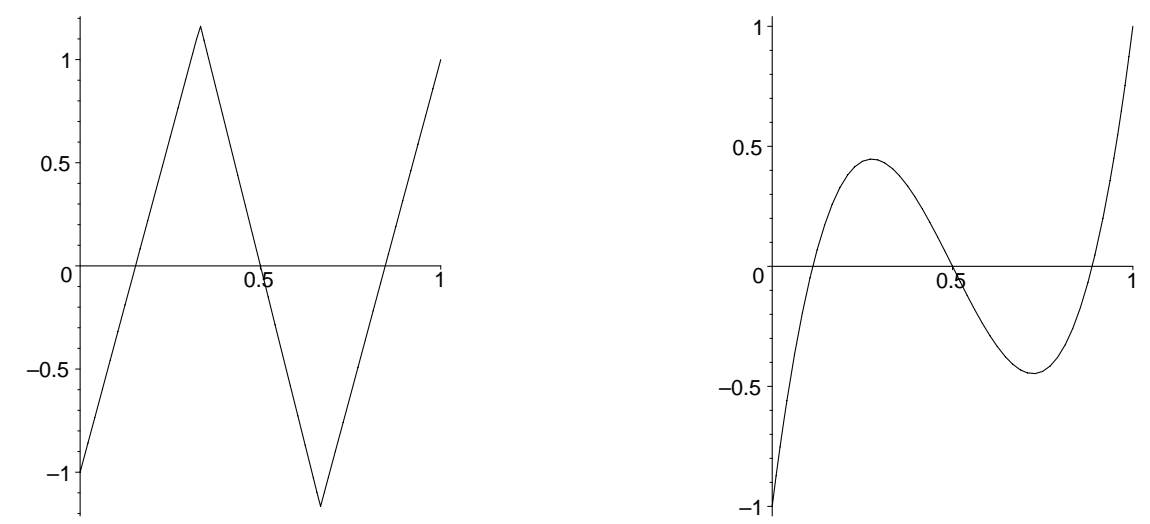

Figure 2.4. Piecewise linear (left) and cubic (right) function $g$.
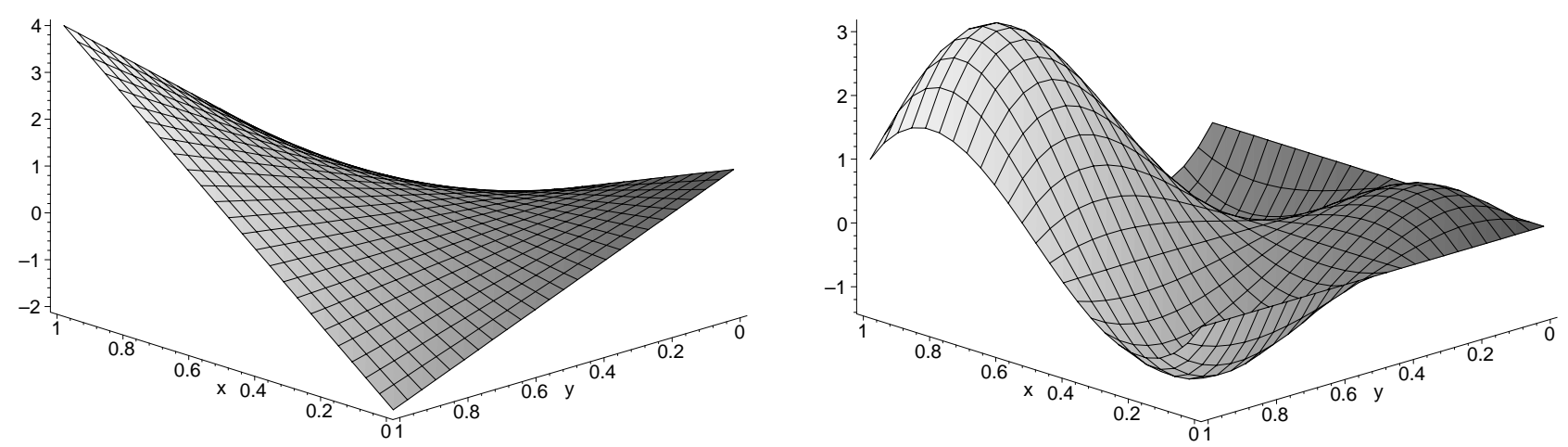

Figure 2.5. Dual bilinear (left) and dual bicubic basis (right), (hexahedral).

functions as a product of the one dimensional modified dual basis functions. For simplicity, we restrict ourselves to the case of piecewise bicubic dual basis functions. We note that the support of the modified dual basis functions $\mu_{p}$ associated with the interior vertex $p$ contains four elements. Moreover, we find $\operatorname{supp} \hat{\mu}_{p}=\operatorname{supp} \varphi_{p}^{s}$, where $\varphi_{p}^{s}$ are the standard nodal basis functions on the slave side.

Figure 2.5 illustrates locally the correction $g$ and the dual basis functions associated with the corner $(1,1)$. Here, we assume that the reference element $(0,1)^{2}$ does not touch the boundary of the interface. On the left, the piecewise bilinear dual basis function is depicted. It is discontinuous with values $4,-2$ and 1 at the vertices. On the boundary of the support, it has values in $[-2,1]$. Adding the bicubic correction, we obtain a continuous bicubic dual basis function which is shown in the right picture of Figure 2.5. The correction has the values -3 , 2 and -1 at the vertices. Our new dual basis functions $\hat{\mu}_{p}$ satisfy $\hat{\mu}_{p}\left(p^{\prime}\right)=\delta_{p, p^{\prime}}$ for all interior vertices $p$ and $p^{\prime}$ of the interface.

Finally, we consider the situation of a simplicial triangulation. We introduce our new dual basis function $\hat{\mu}_{p}$ associated with the interior vertex $p$ as a modification of the dual basis function discussed in [14]. In contrast to the hexahedral case, it is not possible to satisfy supp $\hat{\mu}_{p}=\operatorname{supp} \varphi_{p}^{s}$ for a continuous dual basis function without losing the optimality of the corresponding mortar method. The a priori error analysis requires that the constants are contained in the Lagrange multiplier space. Assuming supp $\hat{\mu}_{p}=\operatorname{supp} \varphi_{p}^{s}$, we find that $\hat{\mu}_{p}(q)=0$ for all vertices $q$ on $\partial \gamma_{m}$ having zero or at least two interior edges.

In the left picture of Figure 2.6, the boundary vertices $q$ such that $\hat{\mu}_{p}(q)=0$ for all interior vertices $p$ are marked with a filled circle. Thus, we cannot guarantee continuity of $\hat{\mu}_{p}$ and supp $\hat{\mu}_{p}=\operatorname{supp} \varphi_{p}^{s}$ at the same 

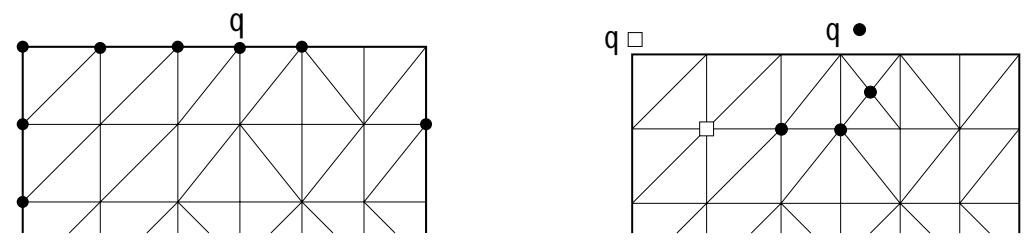

FiguRE 2.6. Boundary vertices with zero value (left) and face groups (right).
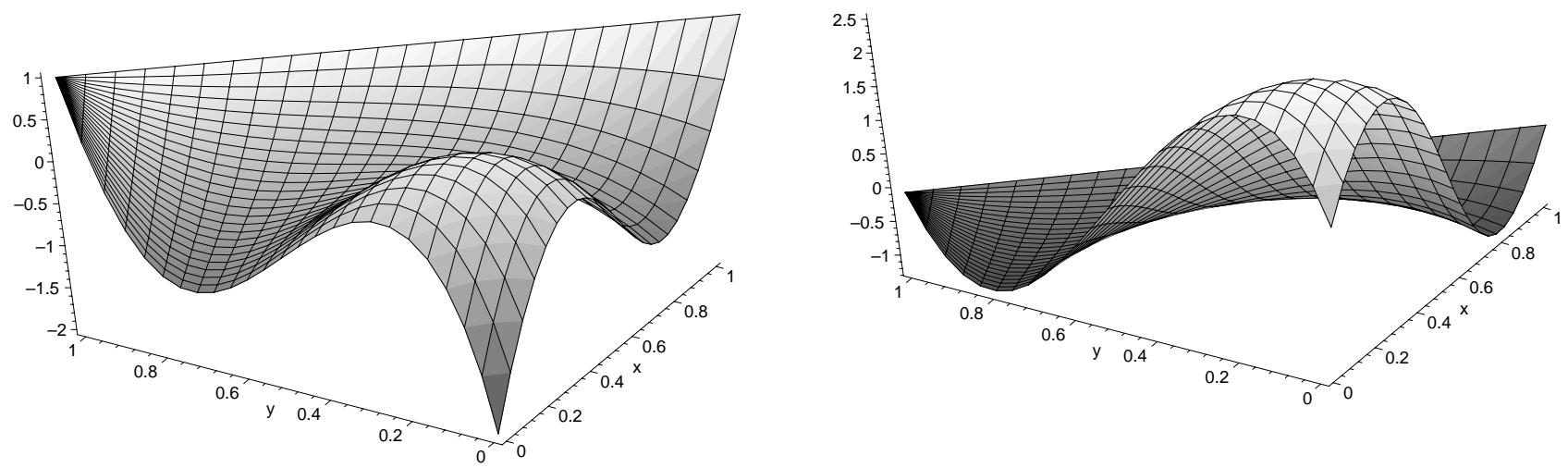

Figure 2.7. Correction $g_{p}$ (left) and modified cubic dual basis $d_{F ; p}$ (right), (simplicial).

time. For each face $F$ on $\gamma_{m}$, we define locally three dual basis functions associated with the three vertices $p_{1}$, $p_{2}, p_{3}$

$$
d_{F ; p_{i}}:=3 \lambda_{p_{i}}-\lambda_{p_{(i+1) \bmod 3}}-\lambda_{p_{(i+2) \bmod 3}}+g_{p_{i}}
$$

where $\lambda_{p_{i}}, 1 \leq i \leq 3$, are the barycentric coordinate functions associated with the three vertices of $F$, respectively. The correction $g_{p_{i}}$ is a cubic function and given by:

$$
g_{p_{i}}:=\left(1-3 \lambda_{p_{i}}\right)^{2}\left(1-\frac{3}{2} \lambda_{p_{i}}\right)-\frac{3}{2} \lambda_{p_{i}}\left(1-\lambda_{p_{i}}\right)\left(7-11 \lambda_{p_{i}}\right)+30 \lambda_{p_{1}} \lambda_{p_{2}} \lambda_{p_{3}} .
$$

Figure 2.7 illustrates locally the correction $g_{p}$ and the dual basis function $d_{F ; p}$ associated with the reference element and the vertex $p=(0,0)$.

Before we define our modified dual basis function, we associate with each vertex in $\bar{\gamma}_{m}$ a continuous dual basis function $\tilde{\mu}_{p}$. It is defined locally in terms of $d_{F ; p_{i}}$

$$
\tilde{\mu}_{p_{\left.\right|_{F}}}:= \begin{cases}d_{F ; p}, & p \text { vertex of } F \\ 0, & \text { elsewhere. }\end{cases}
$$

Then, it is easy to see that $\tilde{\mu}_{p}$ is continuous and satisfies $\int_{\gamma_{m}} \tilde{\mu}_{p} \varphi_{q}^{s} \mathrm{~d} \sigma=\delta_{p q} \int_{\gamma_{m}} \varphi_{q}^{s} \mathrm{~d} \sigma$. Moreover, we find $\sum_{p \in \mathcal{P}_{m}} \tilde{\mu}_{p}=1$. Here, $\mathcal{P}_{m}$ denotes the set of vertices on $\bar{\gamma}_{m}$. Unfortunately due to the discrete inf-sup condition in the analysis of the mortar setting, we have to reduce the degrees of freedom for the Lagrange multiplier space. This can be done by removing the degrees of freedom which are associated with the vertices on $\partial \gamma_{m}$. Let us define the set of the interior vertices by $\mathcal{P}_{m}^{I}$ and the vertices on the boundary by $\mathcal{P}_{m}^{B}$. For each vertex $q \in \mathcal{P}_{m}^{B}$ we define a non-empty set $\mathcal{P}_{q} \subset \mathcal{P}_{m}^{I}$. A vertex $p \in \mathcal{P}_{m}^{I}$ is an element of $\mathcal{P}_{q}$ if and only if $p$ and $q$ are vertices of one face $F$ or there exists two adjacent faces $F_{p}$ and $F_{q}$ such that $p$ vertex of $F_{p}$, $q$ vertex of $F_{q}$ and all the 
vertices of $F_{q}$ are elements of $\mathcal{P}_{m}^{B}$. The set $\mathcal{P}_{q}$ is illustrated for two different choices of $q$ in the right picture of Figure 2.6.

Now, we define for each $p \in \mathcal{P}_{m}^{I}$ our modified dual basis function by

$$
\hat{\mu}_{p}:=\tilde{\mu}_{p}+\sum_{q \in \mathcal{P}_{m}^{B}} \alpha_{p q} \tilde{\mu}_{q},
$$

where the weights are given by $\alpha_{p q}=0$ if $p \notin \mathcal{P}_{q}$ and $\alpha_{p q}=n_{q}^{-1}$. Here, $n_{q}$ is the number of elements in $\mathcal{P}_{q}$. We refer also to $[2,4,14]$ for more details. We note that if $\alpha_{p q}=0$ for all $q \in \mathcal{P}_{m}^{B}$, we find $\operatorname{supp} \hat{\mu}_{p}=\operatorname{supp} \varphi_{p}^{s}$.

Lemma 2.2. The modified basis functions $\hat{\mu}_{p}$ defined by (2.2) are continuous and define a biorthogonal set of basis functions, i.e., $\int_{\gamma_{m}} \hat{\mu}_{p} \varphi_{q}^{s} \mathrm{~d} \sigma=\delta_{p q} \int_{\gamma_{m}} \varphi_{q}^{s} \mathrm{~d} \sigma, p, q \in \mathcal{P}_{m}^{B}$. Moreover, we find

$$
\sum_{p \in \mathcal{P}_{m}^{I}} \hat{\mu}_{p}=1
$$

The proof follows by a straightforward computation.

To obtain upper bounds of the discretization error, we have to analyze the best approximation error, the consistency error and a discrete inf-sup condition. We refer to $[3,5,13]$ for the basic techniques. In particular, the proof of the best approximation property is based on the stability of the mortar projection in the $H_{00}^{1 / 2}$-norm and in a mesh dependent $L^{2}$-norm, respectively. To obtain a optimal discretization scheme, it is sufficient to verify the properties (Sa-Sd) given in [14]. Here, we are working with a biorthogonal set of locally defined Lagrange multiplier basis functions, and by construction the constants are contained in the Lagrange multiplier space. Thus, the conditions ( $\mathrm{Sa}-\mathrm{Sd})$ are automatically satisfied.

Remark 2.3. The introduced modified dual basis functions yield optimal mortar methods. In particular if the solution $u$ is regular enough, the discretization error in the energy norm is of order $h$ and in the $L^{2}$-norm of order $h^{2}$. The Lagrange multiplier can be associated with the $H_{00}^{1 / 2}$-dual norm or with a mesh dependent $L^{2}$-norm. For both norms a priori estimates of $\mathcal{O}(h)$ hold.

\section{NumericAl RESUlts}

Here, we present some numerical examples which illustrate the flexibility and efficiency of the mortar finite element method with dual Lagrange multipliers. The implementation is based on the finite element toolbox ug [1]. We do not discuss and analyze an iterative solver for the arising linear system. In the experiments reported here, we have used a multigrid method for a positive definite system on the product space as our iterative solver. This approach has been applied in [12], and further analyzed in [9, 14]. We present some numerical results in 2D and 3D illustrating the discretization errors for the different Lagrange multipliers. We denote by $u_{h}^{l}, u_{h}^{b}$, and $u_{h}^{c}$ the mortar finite element solutions associated with the different Lagrange multiplier spaces $M_{h}^{l}, M_{h}^{b}$ and $M_{h}^{c}$, respectively. We recall that the definition of $M_{h}^{l}$ is based on the discontinuous piecewise linear shifted hat functions. The spaces $M_{h}^{b}$ and $M_{h}^{c}$ are defined in terms of dual and continuous basis functions. We refer to Section 2 for the exact definition. In the case of $M_{h}^{b}$, the basis functions are piecewise linear and each edge is decomposed into three subedges, whereas the basis functions of $M_{h}^{c}$ are piecewise cubic. We use only uniform refinement. Starting with an initial triangulation $\mathcal{T}_{0}$, the triangulation $\mathcal{T}_{l}$ on level $l$ is obtained by uniform refinement of $\mathcal{T}_{l-1}$.

The different Lagrange multiplier spaces are compared for the following examples. Example 1 is given by: $-\Delta u=f$ on $(0,1)^{2}$, where the right hand side $f$ and the Dirichlet boundary conditions are chosen so that the exact solution is $u(x, y)=\sin (2 \pi y) \exp \left(-x^{-2}\right) \exp \left(-0.1(1-x)^{-2}\right)+\sin (\pi x) \exp \left(-1.25 y^{-2}\right) \exp \left(-0.1(1-y)^{-2}\right)$. The isolines of the solution and the initial triangulation are given in Figure 3.1. The domain is decomposed into six subdomains, defined by $\Omega_{i j}:=((i-1) / 3, i / 3) \times((j-1) / 2, j / 2), 1 \leq i \leq 3,1 \leq j \leq 2$, and the 

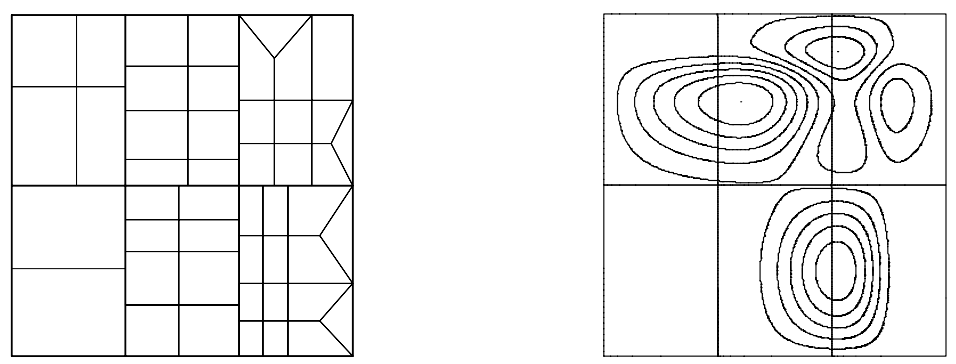

Figure 3.1. Decomposition into 6 subdomains and initial triangulation (left) and isolines of the solution (right), (example 1).

TABle 3.1. Discretization errors in the $L^{2}$-norm (example 1).

\begin{tabular}{|c|c|c|c|c|}
\hline level & $\#$ elem. & $\left\|u-u_{h}^{l}\right\|_{0}$ & $\left\|u-u_{h}^{b}\right\|_{0}$ & $\left\|u-u_{h}^{c}\right\|_{0}$ \\
\hline \hline 0 & 47 & $3.556083 \mathrm{e}-01$ & $3.570347 \mathrm{e}-01$ & $3.564361 \mathrm{e}-01$ \\
\hline 1 & 188 & $1.030630 \mathrm{e}-01$ & $1.036853 \mathrm{e}-01$ & $1.033839 \mathrm{e}-01$ \\
\hline 2 & 752 & $2.618469 \mathrm{e}-02$ & $2.620788 \mathrm{e}-02$ & $2.620249 \mathrm{e}-02$ \\
\hline 3 & 3008 & $6.668651 \mathrm{e}-03$ & $6.686619 \mathrm{e}-03$ & $6.678540 \mathrm{e}-03$ \\
\hline 4 & 12032 & $1.673186 \mathrm{e}-03$ & $1.675372 \mathrm{e}-03$ & $1.674366 \mathrm{e}-03$ \\
\hline 5 & 48128 & $4.192514 \mathrm{e}-04$ & $4.195200 \mathrm{e}-04$ & $4.193982 \mathrm{e}-04$ \\
\hline 6 & 192512 & $1.047745 \mathrm{e}-04$ & $1.048085 \mathrm{e}-04$ & $1.047931 \mathrm{e}-04$ \\
\hline 7 & 770048 & $2.618466 \mathrm{e}-05$ & $2.618888 \mathrm{e}-05$ & $2.618696 \mathrm{e}-05$ \\
\hline
\end{tabular}

TABLE 3.2. Discretization errors in the energy norm (example 1).

\begin{tabular}{|c|c|c|c|c|}
\hline level & $\#$ elem. & $\left\|u-u_{h}^{l}\right\|_{1}$ & $\left\|u-u_{h}^{b}\right\|_{1}$ & $\left\|u-u_{h}^{c}\right\|_{1}$ \\
\hline \hline 0 & 47 & $6.459581 \mathrm{e}-01$ & $6.468066 \mathrm{e}-01$ & $6.464120 \mathrm{e}-01$ \\
\hline 1 & 188 & $3.583403 \mathrm{e}-01$ & $3.589842 \mathrm{e}-01$ & $3.586862 \mathrm{e}-01$ \\
\hline 2 & 752 & $1.816719 \mathrm{e}-01$ & $1.817745 \mathrm{e}-01$ & $1.817470 \mathrm{e}-01$ \\
\hline 3 & 3008 & $9.311620 \mathrm{e}-02$ & $9.321676 \mathrm{e}-02$ & $9.317245 \mathrm{e}-02$ \\
\hline 4 & 12032 & $4.660106 \mathrm{e}-02$ & $4.662382 \mathrm{e}-02$ & $4.661353 \mathrm{e}-02$ \\
\hline 5 & 48128 & $2.331490 \mathrm{e}-02$ & $2.332092 \mathrm{e}-02$ & $2.331823 \mathrm{e}-02$ \\
\hline 6 & 192512 & $1.165473 \mathrm{e}-02$ & $1.165623 \mathrm{e}-02$ & $1.165556 \mathrm{e}-02$ \\
\hline 7 & 770048 & $5.826501 \mathrm{e}-03$ & $5.826876 \mathrm{e}-03$ & $5.826707 \mathrm{e}-03$ \\
\hline
\end{tabular}

triangulations do not match at the interfaces. We have two interior crosspoints and seven interfaces. The meshes at the interfaces are non-matching and the master sides are chosen randomly. Isolines which are almost parallel and isolines which are almost orthogonal to the interfaces can be observed. 
TABLE 3.3. Discretization errors in the weighted Lagrange multiplier norm (example 1).

\begin{tabular}{|c|c|c|c|c|c|c|}
\hline level & $\left\|\lambda-\lambda_{h}^{l}\right\|_{h}$ & ratio & $\left\|\lambda-\lambda_{h}^{b}\right\|_{h}$ & ratio & $\left\|\lambda-\lambda_{h}^{c}\right\|_{h}$ & ratio \\
\hline \hline 0 & $6.357196 \mathrm{e}-02$ & & $5.429382 \mathrm{e}-02$ & & $6.364472 \mathrm{e}-02$ & \\
\hline 1 & $1.700410 \mathrm{e}-02$ & 0.267477988 & $1.407872 \mathrm{e}-02$ & 0.259306123 & $1.726847 \mathrm{e}-02$ & 0.271326042 \\
\hline 2 & $6.984458 \mathrm{e}-03$ & 0.410751407 & $5.505231 \mathrm{e}-03$ & 0.391032068 & $7.090888 \mathrm{e}-03$ & 0.410626303 \\
\hline 3 & $2.604416 \mathrm{e}-03$ & 0.372887345 & $2.132025 \mathrm{e}-03$ & 0.387272577 & $2.706801 \mathrm{e}-03$ & 0.381729481 \\
\hline 4 & $7.401120 \mathrm{e}-04$ & 0.284175799 & $6.113596 \mathrm{e}-04$ & 0.286750671 & $7.765137 \mathrm{e}-04$ & 0.286875060 \\
\hline 5 & $2.889462 \mathrm{e}-04$ & 0.390408748 & $2.365562 \mathrm{e}-04$ & 0.386934628 & $3.020297 \mathrm{e}-04$ & 0.388956048 \\
\hline 6 & $9.844796 \mathrm{e}-05$ & 0.340713807 & $8.073766 \mathrm{e}-05$ & 0.341304349 & $1.030921 \mathrm{e}-04$ & 0.341331001 \\
\hline 7 & $3.473005 \mathrm{e}-05$ & 0.352775720 & $2.847319 \mathrm{e}-05$ & 0.352663057 & $3.637401 \mathrm{e}-05$ & 0.352830236 \\
\hline
\end{tabular}

In Table 3.1 and Table 3.2, the discretization errors are given in the $L^{2}$-norm and in the energy norm, respectively. The observed asymptotic rates confirm the theory. We find that the energy error is of order $h$ whereas the error in the $L^{2}$-norm is of order $h^{2}$. There is no significant difference in the accuracy between the different mortar algorithms on any level neither in the $L^{2}$-norm nor in the energy norm. On Level 7, the difference in the accuracy between the best $u_{h}^{l}$ and worst $u_{h}^{b}$ mortar solution is less than $0.02 \%$ in the $L^{2}$-norm and less than $0.01 \%$ in the energy norm. The influence of the choice of the Lagrange multiplier space on the accuracy of the solution is negligible. Even on the initial triangulation where we have only 47 elements, the difference in the accuracy is extremely small.

Table 3.3 shows the error of the Lagrange multiplier at the interface. Here, we use a mesh dependent $L^{2}$-norm

$$
\left\|\lambda-\lambda_{h}\right\|_{h}^{2}:=\sum_{m=1}^{M} \sum_{e \in \mathcal{S}_{m ; h_{m}}} h_{e}\left\|\lambda-\lambda_{h}\right\|_{0 ; e}^{2}
$$

where $h_{e}$ is the length of the edge $e$. We recall that from the theoretical point of view, we can expect that the error is of order $h$. In Columns 3, 5 and 7 of Table 3.3, we give the ratio between the error on Level $l$ and Level $l-1,1 \leq l \leq 7$. From the theoretical point of view, we expect asymptotically a ratio which is close to 0.5 . However, our numerical results give values close to 0.35 which corresponds to an order $h^{3 / 2}$. The a priori analysis for the Lagrange multiplier includes two terms. The first one is the best approximation property of the Lagrange multiplier space which is of order $h^{3 / 2}$. Unfortunately, the second term involves the energy norm of the error $u-u_{h}$ which is only of order $h$. However, the error $u-u_{h}$ has only to be taken into account on a small strip of width $h$ on the slave side. Under the assumption that the error in the energy norm is equilibrated, we find that the error on the strip is bounded by $C h^{1 / 2}$ times the error on the slave subdomain. As a consequence, a superconvergence result for the Lagrange multiplier can be established.

In our second example, we consider a decomposition of the unit square $(-0.5,0.5)^{2}$ into three subdomains. Two of the subdomains are non-convex. Figure 3.2 shows the decomposition into subdomains, the non-matching triangulations and the isolines of the solution. The slave sides are defined to be on the middle subdomain. The right hand side $f$ and the Dirichlet boundary conditions of $-\Delta u=f$ are chosen such that the exact solution is given by $x(x-y) \exp \left(-10\left(x^{2}+0.6 y^{2}\right)\right)$.

The discretization errors are given in Tables 3.4, 3.5 and 3.6. As in Example 1, the discretization errors for the different Lagrange multiplier spaces are comparable in the energy norm and the $L^{2}$-norm . We remark that the theory does not make any statements about the constants in the a priori estimates. Our numerical results show that the discretization errors for the different Lagrange multipliers are almost the same. If we consider the 

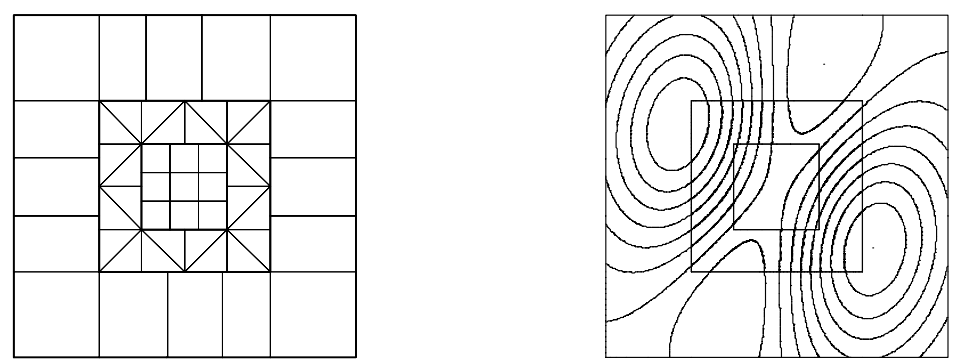

Figure 3.2. Decomposition into 3 subdomains and initial triangulation (left) and isolines of the solution (right), (example 2).

TABLE 3.4. Discretization errors in the $L^{2}$-norm (example 2).

\begin{tabular}{|c|c|c|c|c|}
\hline level & $\#$ elem. & $\left\|u-u_{h}^{l}\right\|_{0}$ & $\left\|u-u_{h}^{b}\right\|_{0}$ & $\left\|u-u_{h}^{c}\right\|_{0}$ \\
\hline \hline 0 & 49 & $1.341098 \mathrm{e}-01$ & $1.335976 \mathrm{e}-01$ & $1.338420 \mathrm{e}-01$ \\
\hline 1 & 196 & $3.723275 \mathrm{e}-02$ & $3.720200 \mathrm{e}-02$ & $3.722088 \mathrm{e}-02$ \\
\hline 2 & 784 & $9.480792 \mathrm{e}-03$ & $9.480434 \mathrm{e}-03$ & $9.480499 \mathrm{e}-03$ \\
\hline 3 & 3136 & $2.383286 \mathrm{e}-03$ & $2.384079 \mathrm{e}-03$ & $2.383755 \mathrm{e}-03$ \\
\hline 4 & 12544 & $5.962056 \mathrm{e}-04$ & $5.962856 \mathrm{e}-04$ & $5.962500 \mathrm{e}-04$ \\
\hline 5 & 50176 & $1.490644 \mathrm{e}-04$ & $1.490747 \mathrm{e}-04$ & $1.490703 \mathrm{e}-04$ \\
\hline 6 & 200704 & $3.726581 \mathrm{e}-05$ & $3.726720 \mathrm{e}-05$ & $3.726661 \mathrm{e}-05$ \\
\hline 7 & 802816 & $9.316213 \mathrm{e}-06$ & $9.316392 \mathrm{e}-06$ & $9.316316 \mathrm{e}-06$ \\
\hline
\end{tabular}

TABLE 3.5. Discretization errors in the energy norm (example 2).

\begin{tabular}{|c|c|c|c|c|}
\hline level & $\#$ elem. & $\left\|u-u_{h}^{l}\right\|_{1}$ & $\left\|u-u_{h}^{b}\right\|_{1}$ & $\left\|u-u_{h}^{c}\right\|_{1}$ \\
\hline \hline 0 & 49 & $4.146493 \mathrm{e}-01$ & $4.144824 \mathrm{e}-01$ & $4.145429 \mathrm{e}-01$ \\
\hline 1 & 196 & $2.146955 \mathrm{e}-01$ & $2.148674 \mathrm{e}-01$ & $2.147914 \mathrm{e}-01$ \\
\hline 2 & 784 & $1.079191 \mathrm{e}-01$ & $1.079724 \mathrm{e}-01$ & $1.079451 \mathrm{e}-01$ \\
\hline 3 & 3136 & $5.408926 \mathrm{e}-02$ & $5.411218 \mathrm{e}-02$ & $5.410120 \mathrm{e}-02$ \\
\hline 4 & 12544 & $2.705186 \mathrm{e}-02$ & $2.705647 \mathrm{e}-02$ & $2.705413 \mathrm{e}-02$ \\
\hline 5 & 50176 & $1.352673 \mathrm{e}-02$ & $1.352786 \mathrm{e}-02$ & $1.352729 \mathrm{e}-02$ \\
\hline 6 & 200704 & $6.763403 \mathrm{e}-03$ & $6.763696 \mathrm{e}-03$ & $6.763550 \mathrm{e}-03$ \\
\hline 7 & 802816 & $3.381663 \mathrm{e}-03$ & $3.381737 \mathrm{e}-03$ & $3.381700 \mathrm{e}-03$ \\
\hline
\end{tabular}

discretization error for the Lagrange multiplier in more detail, we find that the broken dual Lagrange multiplier gives better results for Examples 1 and 2 . From the beginning the error $\left\|\lambda-\lambda_{h}^{b}\right\|_{h}$ is smaller than $\left\|\lambda-\lambda_{h}^{l}\right\|_{h}$ and $\left\|\lambda-\lambda_{h}^{c}\right\|_{h}$. However, the asymptotic convergence rates are almost the same. 
TABLE 3.6. Discretization errors in the weighted Lagrange multiplier norm (example 2).

\begin{tabular}{|c|c|c|c|c|c|c|}
\hline level & $\left\|\lambda-\lambda_{h}^{l}\right\|_{h}$ & ratio & $\left\|\lambda-\lambda_{h}^{b}\right\|_{h}$ & ratio & $\left\|\lambda-\lambda_{h}^{c}\right\|_{h}$ & ratio \\
\hline \hline 0 & $1.490923 \mathrm{e}-02$ & & $1.305521 \mathrm{e}-02$ & & $1.452221 \mathrm{e}-02$ & \\
\hline 1 & $6.918522 \mathrm{e}-03$ & 0.464042878 & $5.308068 \mathrm{e}-03$ & 0.406586182 & $6.939271 \mathrm{e}-03$ & 0.477838497 \\
\hline 2 & $2.010561 \mathrm{e}-03$ & 0.290605565 & $1.474313 \mathrm{e}-03$ & 0.277749456 & $2.027374 \mathrm{e}-03$ & 0.29215950 \\
\hline 3 & $7.425828 \mathrm{e}-04$ & 0.369341094 & $5.808013 \mathrm{e}-04$ & 0.393947079 & $7.741401 \mathrm{e}-04$ & 0.381843754 \\
\hline 4 & $2.451539 \mathrm{e}-04$ & 0.330136787 & $1.861079 \mathrm{e}-04$ & 0.320432994 & $2.534634 \mathrm{e}-04$ & 0.327412828 \\
\hline 5 & $8.325218 \mathrm{e}-05$ & 0.339591497 & $6.272523 \mathrm{e}-05$ & 0.337036901 & $8.623942 \mathrm{e}-05$ & 0.340244074 \\
\hline 6 & $2.941276 \mathrm{e}-05$ & 0.353297174 & $2.221586 \mathrm{e}-05$ & 0.354177417 & $3.055020 \mathrm{e}-05$ & 0.354248671 \\
\hline 7 & $1.036356 \mathrm{e}-05$ & 0.352349116 & $7.837648 \mathrm{e}-06$ & 0.352795165 & $1.077511 \mathrm{e}-05$ & 0.352701782 \\
\hline
\end{tabular}
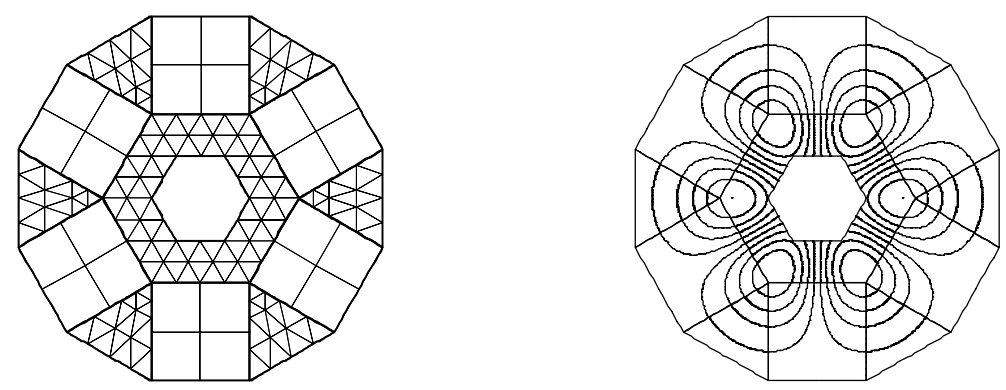

FiguRE 3.3. Decomposition into 13 subdomains and initial triangulation (left) and isolines of the solution (right), (example 3).

In our third example, we consider a decomposition of a non-convex domain into 13 subdomains. Figure 3.3 shows the decomposition into subdomains, the non-matching triangulations and the isolines of the solution. We have 18 interfaces and six crosspoints, each of it shares four subdomains. The master sides are defined to be on the squares. The right hand side $f$ and the Dirichlet boundary conditions of $-\Delta u=f$ are chosen such that the exact solution is given by $x\left(x^{2}-3 y^{2}\right) \exp \left(-2\left(x^{2}+y^{2}\right)\right)$. We combine linear finite elements on triangles with bilinear finite elements on rectangles.

Tables 3.7 and 3.8 give the discretization errors in the $L^{2}$-norm and the energy norm. Asymptotically, we observe the predicted orders. As in Examples 1 and 2, the error in the mesh dependent Lagrange multiplier norm shows a superconvergence behavior. Again the broken dual Lagrange multiplier space yields on the final level better results. Only on Level 1, a considerably better error reduction can be observed. On all other refinement levels, the error reduction factors are approximately the same.

In our last 2D example, we consider the influence of the Lagrange multiplier space in more detail. To do so, we restrict ourselves to a decomposition of the unit square into two rectangles $\Omega_{1}:=(0,1) \times(0,0.5)$ and $\Omega_{2}:=(0,1) \times(0.5,1)$. The right hand side $f$ and the boundary conditions of $-\Delta u=f$ are chosen such that the exact solution is given by $\cos \left(50\left(x-\frac{1}{2}\right) y\right)(1-x)$. Then, the discrete Lagrange multiplier is an approximation of the flux $\lambda(x)=50 \sin \left(25\left(x-\frac{1}{2}\right)\right)(1-x)\left(x-\frac{1}{2}\right)$ which is given as a function of $x$. Here, we have fixed the normal direction $n=(0,1)^{T}$. The Lagrange multiplier is a highly varying function along the interface. Figure 3.4 shows the decomposition into subdomains, the non-matching triangulations, the isolines of the solution and the Lagrange multiplier at the interface. The initial triangulation has 42 elements and is 
TABLE 3.7. Discretization errors in the $L^{2}$-norm (example 3 ).

\begin{tabular}{|c|c|c|c|c|}
\hline level & $\#$ elem. & $\left\|u-u_{h}^{l}\right\|_{0}$ & $\left\|u-u_{h}^{b}\right\|_{0}$ & $\left\|u-u_{h}^{c}\right\|_{0}$ \\
\hline \hline 0 & 102 & $2.467606 \mathrm{e}-01$ & $2.467363 \mathrm{e}-01$ & $2.467363 \mathrm{e}-01$ \\
\hline 1 & 408 & $5.995386 \mathrm{e}-02$ & $5.971488 \mathrm{e}-02$ & $5.971488 \mathrm{e}-02$ \\
\hline 2 & 1632 & $1.542444 \mathrm{e}-02$ & $1.539500 \mathrm{e}-02$ & $1.539500 \mathrm{e}-02$ \\
\hline 3 & 6528 & $3.888708 \mathrm{e}-03$ & $3.889202 \mathrm{e}-03$ & $3.889202 \mathrm{e}-03$ \\
\hline 4 & 26112 & $9.734666 \mathrm{e}-04$ & $9.734491 \mathrm{e}-04$ & $9.734491 \mathrm{e}-04$ \\
\hline 5 & 104448 & $2.434654 \mathrm{e}-04$ & $2.434756 \mathrm{e}-04$ & $2.434756 \mathrm{e}-04$ \\
\hline 6 & 417792 & $6.086807 \mathrm{e}-05$ & $6.086894 \mathrm{e}-05$ & $6.086894 \mathrm{e}-05$ \\
\hline 7 & 1671168 & $1.521698 \mathrm{e}-05$ & $1.521713 \mathrm{e}-05$ & $1.521713 \mathrm{e}-05$ \\
\hline
\end{tabular}

TABLE 3.8. Discretization errors in the energy norm (example 3).

\begin{tabular}{|c|c|c|c|c|}
\hline level & $\#$ elem. & $\left\|u-u_{h}^{l}\right\|_{1}$ & $\left\|u-u_{h}^{b}\right\|_{1}$ & $\left\|u-u_{h}^{c}\right\|_{1}$ \\
\hline \hline 0 & 102 & $4.711325 \mathrm{e}-01$ & $4.711626 \mathrm{e}-01$ & $4.711626 \mathrm{e}-01$ \\
\hline 1 & 408 & $2.097535 \mathrm{e}-01$ & $2.100766 \mathrm{e}-01$ & $2.100766 \mathrm{e}-01$ \\
\hline 2 & 1632 & $1.052478 \mathrm{e}-01$ & $1.052659 \mathrm{e}-01$ & $1.052659 \mathrm{e}-01$ \\
\hline 3 & 6528 & $5.271760 \mathrm{e}-02$ & $5.272434 \mathrm{e}-02$ & $5.272434 \mathrm{e}-02$ \\
\hline 4 & 26112 & $2.636564 \mathrm{e}-02$ & $2.636728 \mathrm{e}-02$ & $2.636728 \mathrm{e}-02$ \\
\hline 5 & 104448 & $1.318236 \mathrm{e}-02$ & $1.318292 \mathrm{e}-02$ & $1.318292 \mathrm{e}-02$ \\
\hline 6 & 417792 & $6.590890 \mathrm{e}-03$ & $6.591026 \mathrm{e}-03$ & $6.591026 \mathrm{e}-03$ \\
\hline 7 & 1671168 & $3.295348 \mathrm{e}-03$ & $3.295382 \mathrm{e}-03$ & $3.295382 \mathrm{e}-03$ \\
\hline
\end{tabular}
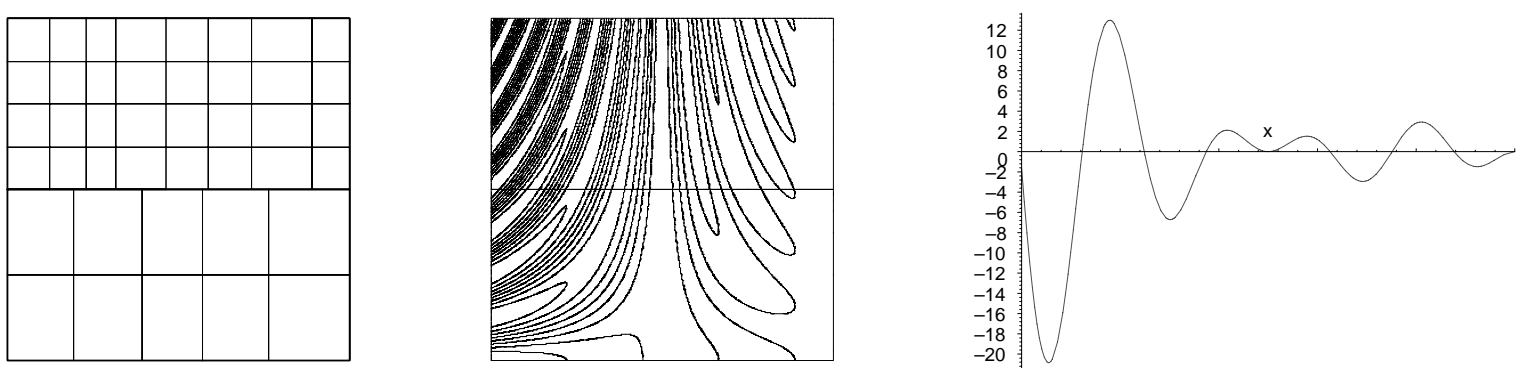

Figure 3.4. Decomposition into 2 subdomains and initial triangulation (left), isolines of the solution (middle) and Lagrange multiplier at the interface (right), (example 4).

non-matching at the interface. Uniform refinement is applied in each refinement step. We choose the slave side to be associated with the lower subdomain. Thus on the initial triangulation, we have four degrees of freedom for the discrete Lagrange multiplier. 
TABLE 3.9. Discretization errors in the weighted Lagrange multiplier norm (example 3).

\begin{tabular}{|c|c|c|c|c|c|c|}
\hline level & $\left\|\lambda-\lambda_{h}^{l}\right\|_{h}$ & ratio & $\left\|\lambda-\lambda_{h}^{b}\right\|_{h}$ & ratio & $\left\|\lambda-\lambda_{h}^{c}\right\|_{h}$ & ratio \\
\hline \hline 0 & $8.681348 \mathrm{e}-02$ & & $8.667644 \mathrm{e}-02$ & & $8.679863 \mathrm{e}-02$ & \\
\hline 1 & $4.495296 \mathrm{e}-02$ & 0.517810828 & $3.289342 \mathrm{e}-02$ & 0.379496665 & $4.541886 \mathrm{e}-02$ & 0.523267014 \\
\hline 2 & $1.644077 \mathrm{e}-02$ & 0.365732757 & $1.159120 \mathrm{e}-02$ & 0.352386586 & $1.647677 \mathrm{e}-02$ & 0.362773746 \\
\hline 3 & $5.918317 \mathrm{e}-03$ & 0.359978091 & $4.125440 \mathrm{e}-03$ & 0.355911381 & $5.948153 \mathrm{e}-03$ & 0.361002368 \\
\hline 4 & $2.119629 \mathrm{e}-03$ & 0.358147257 & $1.467005 \mathrm{e}-03$ & 0.355599645 & $2.133504 \mathrm{e}-03$ & 0.358683443 \\
\hline 5 & $7.496224 \mathrm{e}-04$ & 0.353657361 & $5.207748 \mathrm{e}-04$ & 0.354991837 & $7.576485 \mathrm{e}-04$ & 0.355119324 \\
\hline 6 & $2.652300 \mathrm{e}-04$ & 0.353818135 & $1.840255 \mathrm{e}-04$ & 0.353368672 & $2.682630 \mathrm{e}-04$ & 0.354073161 \\
\hline 7 & $9.380096 \mathrm{e}-05$ & 0.353658937 & $6.513715 \mathrm{e}-05$ & 0.353957196 & $9.495799 \mathrm{e}-05$ & 0.353973488 \\
\hline
\end{tabular}

TABLE 3.10. Discretization errors (exact integration), (example 4).

\begin{tabular}{|c|c|c|c|c|c|c|}
\hline & \multicolumn{2}{|c|}{$L^{2}$-norm } & \multicolumn{2}{c|}{ energy norm } & \multicolumn{2}{c|}{ weighted LM norm } \\
\hline level & $\left\|u-u_{h}^{l}\right\|_{0}$ & $\left\|u-u_{h}^{c}\right\|_{0}$ & $\left\|u-u_{h}^{l}\right\|_{1}$ & $\left\|u-u_{h}^{c}\right\|_{1}$ & $\left\|\lambda-\lambda_{h}^{l}\right\|_{h}$ & $\left\|\lambda-\lambda_{h}^{c}\right\|_{h}$ \\
\hline \hline 0 & $1.566184 \mathrm{e}+00$ & $1.564561 \mathrm{e}+00$ & $1.022139 \mathrm{e}+00$ & $1.021536 \mathrm{e}+00$ & $2.572471 \mathrm{e}+00$ & $2.572471 \mathrm{e}+00$ \\
\hline 1 & $3.877635 \mathrm{e}-01$ & $3.867007 \mathrm{e}-01$ & $6.425701 \mathrm{e}-01$ & $6.427550 \mathrm{e}-01$ & $1.024982 \mathrm{e}+00$ & $1.024982 \mathrm{e}+00$ \\
\hline 2 & $1.087264 \mathrm{e}-01$ & $1.087453 \mathrm{e}-01$ & $3.282757 \mathrm{e}-01$ & $3.283115 \mathrm{e}-01$ & $6.150985 \mathrm{e}-01$ & $6.150985 \mathrm{e}-01$ \\
\hline 3 & $2.815036 \mathrm{e}-02$ & $2.813904 \mathrm{e}-02$ & $1.666804 \mathrm{e}-01$ & $1.667178 \mathrm{e}-01$ & $2.615208 \mathrm{e}-01$ & $2.615208 \mathrm{e}-01$ \\
\hline 4 & $7.098702 \mathrm{e}-03$ & $7.097746 \mathrm{e}-03$ & $8.363201 \mathrm{e}-02$ & $8.364393 \mathrm{e}-02$ & $9.573677 \mathrm{e}-02$ & $9.573677 \mathrm{e}-02$ \\
\hline 5 & $1.778255 \mathrm{e}-03$ & $1.778188 \mathrm{e}-03$ & $4.184923 \mathrm{e}-02$ & $4.185135 \mathrm{e}-02$ & $3.388482 \mathrm{e}-02$ & $3.388482 \mathrm{e}-02$ \\
\hline 6 & $4.447739 \mathrm{e}-04$ & $4.447664 \mathrm{e}-04$ & $2.092873 \mathrm{e}-02$ & $2.092929 \mathrm{e}-02$ & $1.193008 \mathrm{e}-02$ & $1.193008 \mathrm{e}-02$ \\
\hline 7 & $1.112068 \mathrm{e}-04$ & $1.112059 \mathrm{e}-04$ & $1.046482 \mathrm{e}-02$ & $1.046497 \mathrm{e}-02$ & $4.204846 \mathrm{e}-03$ & $4.204846 \mathrm{e}-03$ \\
\hline
\end{tabular}

In this example, we are interested in the influence of the Lagrange multiplier space if quadrature rules are applied. We compare the piecewise linear but discontinuous with the piecewise cubic but continuous dual Lagrange multiplier basis functions. Following the ideas in $[7,10]$, we use a non-symmetric Petrov-Galerkin approach. The test space is defined in terms of a weak coupling condition where the exact integral is replaced by a quadrature formula based on the edges of the master side. For the definition of the trial space, a quadrature formula based on the edges of the slave side is used. As a consequence, the resulting stiffness matrix is nonsymmetric. Moreover, we can interpret the mortar setting as follows: On the slave side, we solve a non homogeneous Dirichlet problem. The definition of the trial space yields that the boundary condition are realized in terms of a quadrature formula based on the slave side. On the master side, we solve a non homogeneous Neumann problem. Now, the definition of the test space guarantees that the boundary conditions are realized in terms of a quadrature formula based on the master side. Thus the choice of the quadrature formulas is natural.

In a first step, we compute the discretization errors in the $L^{2}$-norm, the energy norm and the mesh dependent $L^{2}$-norm on the interface if exact integration is applied. The numerical results given in Table 3.10 show that there is no qualitative and quantitative difference in the two dual Lagrange multipliers. 
TABLE 3.11. Discretization errors (quadrature formula), (example 4).

\begin{tabular}{|c|c|c|c|c|c|c|}
\hline & \multicolumn{2}{|c|}{$L^{2}$-norm } & \multicolumn{2}{c|}{ energy norm } & \multicolumn{2}{c|}{ weighted LM norm } \\
\hline level & $\left\|u-u_{h}^{l}\right\|_{0}$ & $\left\|u-u_{h}^{c}\right\|_{0}$ & $\left\|u-u_{h}^{l}\right\|_{1}$ & $\left\|u-u_{h}^{c}\right\|_{1}$ & $\left\|\lambda-\lambda_{h}^{l}\right\|_{h}$ & $\left\|\lambda-\lambda_{h}^{c}\right\|_{h}$ \\
\hline \hline 0 & $1.597459 \mathrm{e}+00$ & $1.590183 \mathrm{e}+00$ & $1.021226 \mathrm{e}+00$ & $1.022896 \mathrm{e}+00$ & $2.425751 \mathrm{e}+00$ & $2.692954 \mathrm{e}+00$ \\
\hline 1 & $3.860353 \mathrm{e}-01$ & $3.891088 \mathrm{e}-01$ & $6.424821 \mathrm{e}-01$ & $6.430641 \mathrm{e}-01$ & $9.907894 \mathrm{e}-01$ & $1.126765 \mathrm{e}+00$ \\
\hline 2 & $1.088178 \mathrm{e}-01$ & $1.087666 \mathrm{e}-01$ & $3.284025 \mathrm{e}-01$ & $3.283159 \mathrm{e}-01$ & $5.858385 \mathrm{e}-01$ & $6.178719 \mathrm{e}-01$ \\
\hline 3 & $2.940616 \mathrm{e}-02$ & $2.813497 \mathrm{e}-02$ & $1.668012 \mathrm{e}-01$ & $1.667166 \mathrm{e}-01$ & $2.799113 \mathrm{e}-01$ & $2.615416 \mathrm{e}-01$ \\
\hline 4 & $9.481763 \mathrm{e}-03$ & $7.133550 \mathrm{e}-03$ & $8.365876 \mathrm{e}-02$ & $8.364368 \mathrm{e}-02$ & $9.649008 \mathrm{e}-02$ & $9.581336 \mathrm{e}-02$ \\
\hline 5 & $2.533997 \mathrm{e}-03$ & $1.788415 \mathrm{e}-03$ & $4.185955 \mathrm{e}-02$ & $4.185134 \mathrm{e}-02$ & $3.391396 \mathrm{e}-02$ & $3.390462 \mathrm{e}-02$ \\
\hline 6 & $6.152243 \mathrm{e}-04$ & $4.485467 \mathrm{e}-04$ & $2.093147 \mathrm{e}-02$ & $2.092929 \mathrm{e}-02$ & $1.224866 \mathrm{e}-02$ & $1.193274 \mathrm{e}-02$ \\
\hline 7 & $2.793155 \mathrm{e}-04$ & $1.114683 \mathrm{e}-04$ & $1.046563 \mathrm{e}-02$ & $1.046496 \mathrm{e}-02$ & $4.277662 \mathrm{e}-03$ & $4.204771 \mathrm{e}-03$ \\
\hline
\end{tabular}
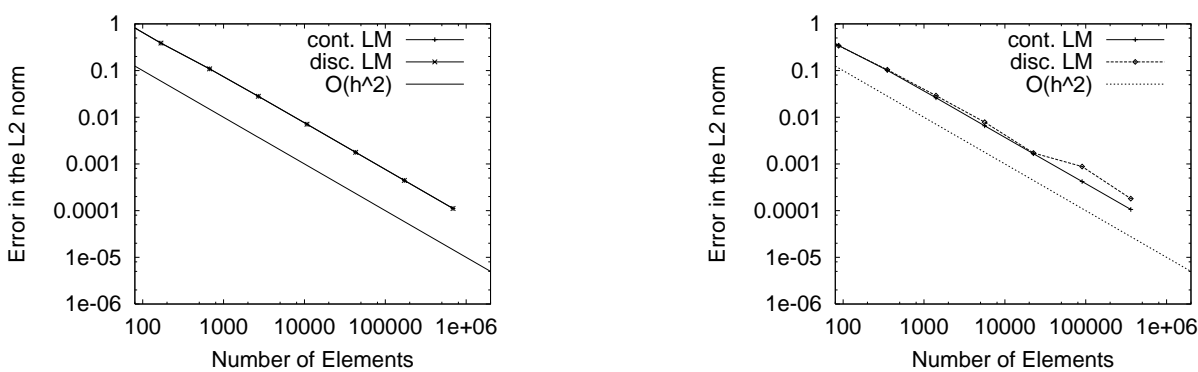

FiguRE 3.5. Error in the $L^{2}$-norm, exact integration (left) and quadrature formulas (right), (example 4).

In a second step, we apply the non-symmetric Petrov-Galerkin approach and work with quadrature rules. The numerical results are given in Table 3.11. Comparing the errors for the two different Lagrange multiplier spaces, we find one significant difference. For the $L^{2}$-norm, the cubic Lagrange multiplier space gives considerably better results than the linear one. In the energy norm and the mesh dependent Lagrange multiplier norm, the difference can be neglected. In the case of cubic Lagrange multipliers, the numerical results obtained by quadrature formulas are as good as if exact integration is used. No loss of accuracy can be observed, see Tables 3.10 and 3.11. This is not the case for the discontinuous piecewise linear Lagrange multiplier space.

In Figure 3.5, we illustrate the numerical results for the $L^{2}$-norm. The solid and the dashed lines represent the numerical results for the continuous and discontinuous dual Lagrange multiplier spaces, respectively.

The straight lines below the numerical results indicate the order $h^{2}$. The left picture shows the discretization error if the constraints are exactly realized. To carry out the exact integration, we use a low order quadrature formula based on the union of vertices on slave and master side. Linear and cubic Lagrange multipliers yield the same qualitative and quantitative results. The situation is different if we use quadrature formulas based on the master side for the test space and based on the slave side for the trial space. Then, the discretization with discontinuous dual basis functions is asymptotically worth than the one obtained with continuous basis functions. On the finest level, a factor of two between the discretization errors can be observed.

Finally, we consider an example in 3D. The domain $(0,1)^{2} \times(0,2.4)$ is decomposed into three subdomains $\Omega_{1}:=(0,1)^{3}, \Omega_{2}:=(0,1)^{2} \times(1,1.4)$, and $\Omega_{3}:=(0,1)^{2} \times(1.4,2.4)$. The right hand side $f$ and the boundary 
solution

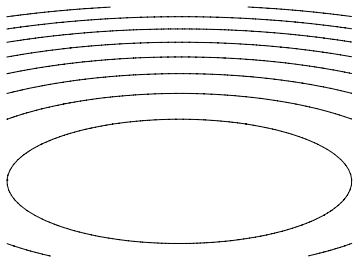

flux

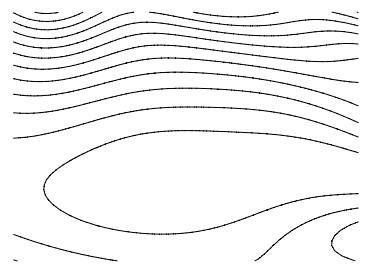

solution

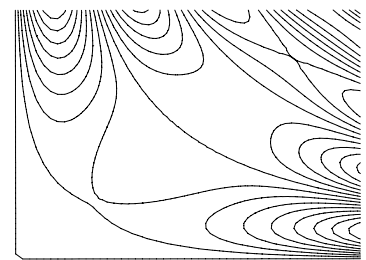

flux

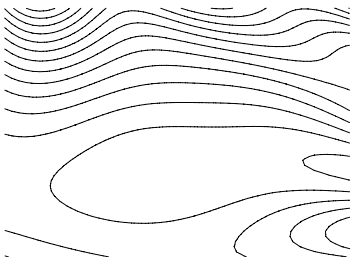

FiguRE 3.6. Isoline of the solution and flux at the lower (left) and upper (right) interface.

TABLE 3.12. Discretization errors in different norms.

\begin{tabular}{|c|c|c|c|c|c|c|}
\hline level & $\left\|u-u_{h}^{c}\right\|_{1}$ & ratio & $\left\|u-u_{h}^{c}\right\|_{0}$ & ratio & $\left\|\lambda-\lambda_{h}^{c}\right\|_{h}$ & ratio \\
\hline \hline 0 & $9.184955 \mathrm{e}-01$ & & $9.270304 \mathrm{e}-01$ & & $1.768651 \mathrm{e}+00$ & \\
\hline 1 & $5.031041 \mathrm{e}-01$ & 0.5477480292 & $2.934020 \mathrm{e}-01$ & 0.3164966326 & $1.193387 \mathrm{e}+00$ & 0.6747441976 \\
\hline 2 & $5.130703 \mathrm{e}-01$ & 1.0198094191 & $1.419080 \mathrm{e}-01$ & 0.4836640513 & $7.110698 \mathrm{e}-01$ & 0.5958417512 \\
\hline 3 & $2.304834 \mathrm{e}-01$ & 0.4492238198 & $3.511381 \mathrm{e}-02$ & 0.2474406657 & $2.675305 \mathrm{e}-01$ & 0.3762366226 \\
\hline 4 & $1.137777 \mathrm{e}-01$ & 0.4936481325 & $9.009302 \mathrm{e}-03$ & 0.2565743221 & $9.965807 \mathrm{e}-02$ & 0.3725110594 \\
\hline 5 & $5.675590 \mathrm{e}-02$ & 0.4988314933 & $2.268062 \mathrm{e}-03$ & 0.2517466946 & $3.563891 \mathrm{e}-02$ & 0.3576118823 \\
\hline
\end{tabular}

conditions of $-\Delta u=f$ are chosen such that the exact solution is given by $u(x, y, z)=5(z-1.4)\left((x-0.5)^{2}+\right.$ $\left.4(y-0.3)^{3}\right)+z(z-1) \sin (4 \pi x y)\left(2(x-y)^{2}+(y+x-1)^{2}\right)$. In Figure 3.6, the trace and flux of the solution at the interfaces are shown. The two pictures on the left and the right illustrate the lower and upper interface, respectively. We start with a very coarse triangulation having 11 elements. The subdomain $\Omega_{2}$ is decomposed into nine hexahedras. In each refinement step we apply uniform refinement and each element is decomposed into eight subelements. On the finest level, we have 360448 elements.

Table 3.12 shows the relative discretization errors in the energy and $L^{2}$-norm as well as the absolute discretization error in the weighted Lagrange multiplier norm. The numerical results are carried out for the continuous piecewise cubic Lagrange multiplier space introduced in Section 2. Asymptotically, we observe the same convergence rates as in $2 \mathrm{D}$. However, the asymptotic phase starts later. This is also related to the fact that we start with a very coarse triangulation. On Level 0 , we have only one element in subdomain $\Omega_{1}$ and $\Omega_{3}$.

\section{REFERENCES}

[1] P. Bastian, K. Birken, K. Johannsen, S. Lang, N. Neuß, H. Rentz-Reichert and C. Wieners, UG - a flexible software toolbox for solving partial differential equations. Comput. Vis. Sci. 1 (1997) 27-40.

[2] D. Braess and W. Dahmen, Stability estimates of the mortar finite element method for 3-dimensional problems. East-West J. Numer. Math. 6 (1998) 249-263.

[3] F. Ben Belgacem, The mortar finite element method with Lagrange multipliers. Numer. Math. 84 (1999) 173-197.

[4] F. Ben Belgacem and Y. Maday, The mortar element method for three dimensional finite elements. RAIRO Modél. Math. Anal. Numér. 31 (1997) 289-302.

[5] C. Bernardi, Y. Maday and A.T. Patera, Domain decomposition by the mortar element method, in: Asymptotic and numerical methods for partial differential equations with critical parameters, H. Kaper et al. Eds., Reidel, Dordrecht (1993) $269-286$.

[6] C. Bernardi, Y. Maday and A.T. Patera, A new nonconforming approach to domain decomposition: the mortar element method, in: Nonlinear partial differential equations and their applications, H. Brezzi et al. Eds., Paris (1994) 13-51.

[7] L. Cazabeau, C. Lacour and Y. Maday, Numerical quadratures and mortar methods, in: Computational science for the 21st century. Dedicated to Prof. Roland Glowinski on the occasion of his 60th birthday. Symposium, Tours, France, May 5-7, 1997, John Wiley \& Sons Ltd. (1997) 119-128. 
[8] C. Kim, R.D. Lazarov, J.E. Pasciak and P.S. Vassilevski, Multiplier spaces for the mortar finite element method in three dimensions. SIAM J. Numer. Anal. 39 (2001) 519-538.

[9] R.H. Krause and B.I. Wohlmuth, Nonconforming domain decomposition techniques for linear elasticity. East-West J. Numer. Math. 8 (2000) 177-206.

[10] Y. Maday, F. Rapetti and B.I. Wohlmuth, The influence of quadrature formulas in 3d mortar methods. Lect. Notes Comput. Sci. Eng. 22, Springer-Verlag (2002).

[11] P. Oswald and B. Wohlmuth, On polynomial reproduction of dual FE bases, in: Thirteenth Int. Conf. on Domain Decomposition Methods (2002) 85-96.

[12] B.I. Wohlmuth and R.H. Krause, Multigrid methods based on the unconstrained product space arising from mortar finite element discretizations. SIAM J. Numer. Anal. 39 (2001) 192-213.

[13] B.I. Wohlmuth, A mortar finite element method using dual spaces for the Lagrange multiplier. SIAM J. Numer. Anal. 38 (2000) 989-1012.

[14] B.I. Wohlmuth, Discretization methods and iterative solvers based on domain decomposition. Lecture Notes in Comput. Sci. 17, Springer, Heidelberg (2001).

To access this journal online:

www.edpsciences.org 